msh-mss Mathématiques et sciences humaines

142 | Été 1998

Varia

\title{
Une introduction élémentaire à l'analyse mathématique des inégalités
}

Elementary introduction to mathematical analysis of inequality

Marc Barbut

\section{(2) OpenEdition}

\section{Journals}

Édition électronique

URL : http://journals.openedition.org/msh/2780

DOI : $10.4000 / \mathrm{msh} .2780$

ISSN : 1950-6821

Éditeur

Centre d'analyse et de mathématique sociales de l'EHESS

Édition imprimée

Date de publication : 1 mars 1998

ISSN : 0987-6936

Référence électronique

Marc Barbut, « Une introduction élémentaire à l'analyse mathématique des inégalités », Mathématiques et sciences humaines [En ligne], 142 | Été 1998, mis en ligne le 10 février 2006, consulté le 23 juillet 2020. URL : http://journals.openedition.org/msh/2780 ; DOI : https://doi.org/10.4000/msh.2780

〔c École des hautes études en sciences sociales 


\title{
UNE INTRODUCTION ELEMENTAIRE A L'ANALYSE MATHEMATIQUE DES INEGALITES'
}

\author{
Marc BARBUT $^{2}$
}

\begin{abstract}
RÉSUMÉ - Le concept d'inégalité (dans une distribution de ressources, de revenus, etc.) est l'un des plus présents dans les esprits, les écrits, les paroles de nos contemporains. Il est pourtant moins facile à appréhender que d'autres catégories psycho-linguistiques telles que la classification ou l'ordre. On présente ici une introduction élémentaire et qui n'a d'autre ambition que pédagogique, aux mathématiques de l'inégalité ; celles-ci pourraient suggérer des thèmes de recherche à la psychologie génétique.
\end{abstract}

SUMMARY - Elementary introduction to mathematical analysis of inequality The concept of inequality (in distributions of wealth, incomes, etc.) is one of the most familiar to contemporaneous minds, in their discourses or writtings. Yet, this concept is less easy to grasp than other psycho-linguistic categories such as classification or ordering. Here is proposed an elementary introduction to pertinent mathematical tools for inequalitie's study; it may suggest some paths for genetic psychology research.

S'il est un phénomène social sujet à discussions souvent passionnées, mais aussi à confusions et malentendus, c'est bien celui des inégalités.

La formalisation mathématique, en dépit de ses simplifications, et avec toutes ses limites, permet une clarification de ces questions.

D'ailleurs, pour comprendre l'acquisition de catégories fondamentales de la pensée, telles par exemple que le classificatoire ou l'ordinal, il est nécessaire (mais non suffisant, bien sûr) de se référer aux formalisations mathématiques correspondantes.

La Psychologie génétique a, dans la voie préconisée par Jean Piaget, consacré de très nombreux travaux à l'étude des processus d'acquisition des deux catégories précitées. Mon intention dans ces quelques pages est donc aussi d'attirer l'attention des psychologues sur ce concept beaucoup moins étudié, sinon totalement ignoré, par la Psychologie génétique, et qui pourtant me semble tout aussi fondamental, parce qu'à la fois présent dans toutes les cervelles et susceptible d'une formalisation mathématique précise : celui d'inégalité.

\footnotetext{
${ }^{1}$ Ce texte, à quelques modifications de détail près, est celui publié sous presque le même titre dans les Archives de Psychologie (Genève, 1990, 58, 99-114), numéro d'hommage à Pierre Gréco, qui venait de disparaître.

Je remercie la direction des Archives de Psychologie et tout particulièrement Madame le Professeur Christiane Gillieron d'en avoir autorisé la réédition.

${ }^{2}$ Centre d'Analyse et de Mathématique Sociales, E.H.E.S.S., 54 boulevard Raspail - 75270 Paris Cedex 06.
} 
Inégalité dans le sens où l'on parle des inégalités économiques ou sociales, ou encore celui dans lequel maint programme politique proclame sa volonté de "réduire les inégalités". Concept étroitement lié à celui de dispersion (ou, dualement, de concentration), puisque qui dit inégalité veut d'abord dire variations, fluctuations par rapport à quelque chose qui serait égal, constant. Formaliser la notion d'inégalité, c'est du même coup formaliser celle de dispersion (concentration) ; si ces deux notions doivent être distinguées, elles ne diffèrent que sur des points non essentiels ${ }^{3}$. Cette formalisation, tient en cinq étapes, et quatre règles ou conditions, règles que les l'on pourra nommer axiomes, si l'on préfère. L'inégalité va être définie comme une relation qui s'énoncera : "être plus ou moins inégalitaire que" et satisfaisant aux conditions que l'on va successivement introduire. Mais de quoi s'agit-il ? Autrement dit : inégalité de quoi ?

\section{PREMIERE ETAPE : LES AFFECTATIONS}

Au départ, il y a, comme toujours lorsque l'on mathématise une situation des applications, ou fonctions (au sens mathématique du terme) : celles que j'appelerai ici des affectations.

Il y a un ensemble B de biens que l'on répartit entre des individus qui constituent un second ensemble I. Chaque bien est attribué à un individu et à un seul ; un individu peut se voir attribuer plusieurs biens, ou un seul, ou éventuellement aucun.

L'affectation, c'est la fonction $\mathrm{f}$ : B 2 I qui à chaque bien fait correspondre l'individu qui le reçoit. Et c'est sur les affectations que va porter la relation d'inégalité.

Les ensembles B et I sont bien entendu finis, d'effectifs $n$ et $k$ respectivement $(\mathrm{PBP}=n, \mathrm{PIP}=k)$.

Remarque : dans certaines théories de la répartition (de la richesse, des revenus, etc...) les ensembles $\mathrm{B}$ et $\mathrm{I}$ sont considérés comme infinis, bien que dans les situations concrètes d'affectation ils soient toujours finis. Le "passage à l'infini" fournit, pour ces théories, des approximations commodes ; ces commodités n'ont rien à voir avec la formalisation de la notion d'inégalité, qui est actuellement mon seul propos.

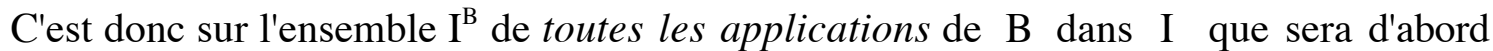
définie (au moyen des règles - ou axiomes - qui vont être énoncés ci-dessous) une relation binaire d'ordre partiel nommée "inégalité" et qui se lira "l'affectation f est au moins aussi inégalitaire que l'affectation $\mathrm{f}^{\prime}$ ".

A ce premier stade, une comparaison encore très grossière de la concentration et de l'inégalité des affectations est déjà possible. Sont considérées comme maximales, dans l'ordre (à construire) d'inégalité (ou de concentration) les affectations dans lesquelles un seul individu reçoit tous les biens : il y en a exactement $k$, autant que d'individus.

Maximales (mais non maximums) en ce sens que pour chacune de ces $k$ affectations, il n'y en a aucune autre que l'on puisse considérer comme plus concentrée qu'elle ; et que toute affectation dans laquelle les biens sont répartis entre plusieurs des individus est moins concentrée, moins inégalitaire, que celles qui attribuent tout à un seul de ces individus : une affectation qui répartit l'ensemble $\mathrm{B}$ des biens entre les deux individus $i, j$ (dont chacun reçoit au moins un bien) est moins inégalitaire que celle qui attribuerait tout à l'individu $i$, et que celle qui attribuerait tout à l'individu $j$. Mais on se gardera de la comparer, au point où nous en sommes, à celle qui attribuerait tout à un troisième individu $h:$ il y faudrait des hypothèses supplémentaires ; ce sont celles-ci que l'on va maintenant introduire.

\footnotetext{
${ }^{3}$ Notamment, la dispersion d'une variable s'exprime dans la même unité de mesure que cette variable ; l'inégalité, par contre, est sans dimension (au sens des physiciens), comme on le verra infra.
} 


\section{DEUXIÈME ETAPE, PREMIERE REGLE : LES PARTITIONS ET LEUR TREILLIS}

Pour que la comparaison que l'on vient d'évoquer ait un sens, on pourrait par exemple considérer que l'individu $h$ est interchangeable avec $i$ et $j$; cela ne va certes pas de soi. En France, par exemple, il faudra attendre 1789 pour que l'on admette, en droit, l'équivalence de tous les individus constituant l'ensemble I dans ces situations typiques de répartition de biens que sont les héritages ; le "droit d'aînesse" de l'Ancien Régime impliquait au contraire leur non équivalence.

Le concept mathématique d'inégalité admet, lui, cette équivalence. C'est la première règle.

RÈGLE (Axiome ) 1. Deux affectations qui se déduisent l'une de l'autre par une permutation des éléments de I ont même inégalité (et même concentration).

Autrement dit : l'inégalité (et la concentration) d'une affectation est invariante par rapport au groupe des permutations de I.

Ce que dit d'abord cette règle, c'est qu'il ne faut surtout pas confondre, comme on le fait trop souvent, le concept mathématique (ou, si l'on préfère, statistique) d'inégalité avec les notions, qui elles sont d'ordre moral, d'équité ou de justice dans une répartition. Pour reprendre un exemple classique, que le chirurgien dont l'art sauve des centaines de vies humaines ait une rémunération vingt fois supérieure à celle du gardien de square est - arithmétiquement - très inégalitaire ; mais cela peut être moralement jugé très équitable, et est bien jugé ainsi en général.

Ce que dit ensuite la première règle, c'est qu'il n'y a à retenir, de chaque affectation, qui est une fonction de B dans I, que la partition (ou la classification) induite sur B par cette fonction : la partition dans laquelle on met dans une même classe tous les éléments de $\mathrm{B}$ qui sont attribués au même individu de I (qui ont la même image dans I). Si dans des affectations distinctes les classes sont les mêmes, si la partition de B est la même, peu importe quel individu reçoit tous les biens de quelle classe : seul compte l'ensemble des classes de biens constituées, la partition.

Or les partitions d'un même ensemble, ici l'ensemble B des biens, ont un ordre (partiel) naturel, qui structure leur ensemble en treillis : une partition $\square$ de B est une sous-partition d'une autre partition $\square^{\prime}$ de B si elle s'en déduit par subdivision des classes de B. On dit encore que $\square$ ' est un affinage de $\square$.

On appelle treillis tout ensemble ordonné tel que, pour tout couple (x,y) de ses éléments, il existe un plus petit de leurs majorants communs, leur supremum, noté $\mathrm{x} ; \mathrm{y}$, et un plus grand de leurs minorants communs, leur infimum, noté $\mathrm{x}: \mathrm{y}$.

Dans le cas des partitions d'un même ensemble ordonnées par subdivision (ou affinage), les classes de l'infimum $\square: \square$ ' de deux quelconques d'entre elles s'obtiennent en prenant l'intersection de chaque classe de la première, $\square$, avec toutes les classes de la seconde, $\square^{\prime}$ : c'est le procédé classique du croisement de deux classifications, celui des tableaux croisés dont sont riches tous les recueils de statistiques.

Quant au supremum, la façon de le construire est moins évidente ; la classe dans $\square$; $\square$ ' d'un élément quelconque $\mathrm{x}$ de l'ensemble partitionné s'obtient en associant à $\mathrm{x}$ tous les éléments de sa classe dans $\square$, puis tous les éléments des classes de ceux-ci dans $\square$ ', puis tous les élément des classes de ces derniers dans $\square$, etc. On en verra un exemple trivial quelques lignes plus loin (sur les partitions d'un ensemble et leur treillis, Cf. [6] par exemple). 
Si l'on désigne par $\mathrm{P}_{\mathrm{n}, \mathrm{r}}$ le nombre des partitions d'un ensemble d'effectif $\mathrm{n}$ en $\mathrm{r}$ classes, on a :

$$
\mathrm{P}_{\mathrm{n}, 1}=\mathrm{P}_{\mathrm{n}, \mathrm{n}}=1 \quad \mathrm{P}_{\mathrm{n}, \mathrm{r}}=0 \quad \text { si } \quad \mathrm{r}>\mathrm{n} .
$$

Les nombres $\mathrm{P}_{\mathrm{n}, \mathrm{r}}$ satisfont à l'équation de récurrence :

$$
P_{n, r}=P_{n-1, r-1}+r P_{n-1, r}
$$

La Figure 1 montre dans des notations évidentes une petite partie du treillis des 52 partitions d'un ensemble $\mathrm{B}=\{\mathrm{a}, \mathrm{b}, \mathrm{c}, \mathrm{d}, \mathrm{e}$,$\} de cinq biens, ordonnées par subdivision. Ainsi, la$ partition la moins fine, (abcde), figuree au sommet du diagramme, correspond à toutes les affectations dans lesquelles un seul individu reçoit tous les biens : c'est la partition la plus inégalitaire. Ses sous-partitions (abcd-e) et (abc-de), non comparables entre elles, peuvent être considérées comme plus égalitaires que (abcde), et leur sous-partition commune (abc-d-e), qui est leur infimum, comme plus égalitaire que toutes les deux. De même, (abc-d-e) et (ab-c-de) sont plus égalitaires que (abc-de), qui est leur supremum. Quant à la partition la plus fine (a-b-cd-e), figurée au bas du diagramme, elle correspond aux affectations les plus égalitaires, celles dans lesquelles chaque individu reçoit un bien. En effet, chaque fois que l'on subdivise une classe d'une partition, cela signifie, pour les affectations correspondantes, qu'on a transféré à un individu qui n'avait rien une partie de ce qu'avait un nanti : il est naturel de poser que l'on a ainsi diminué la concentration et l'inégalité.

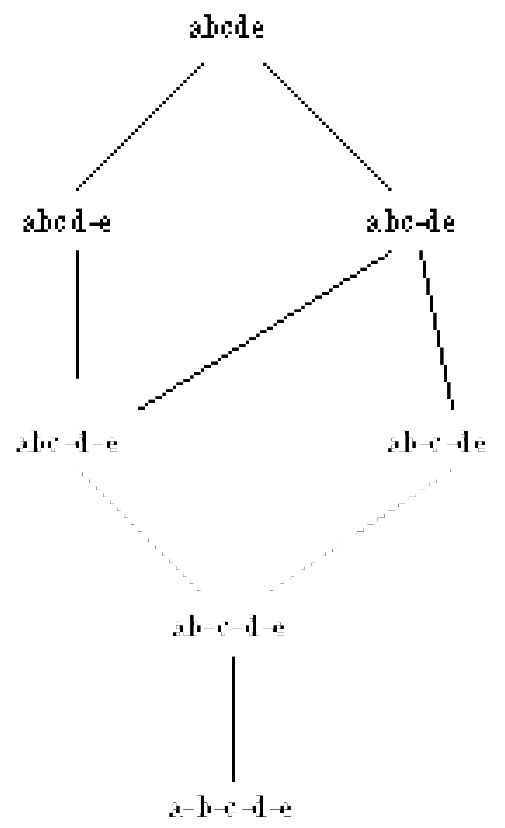

Figure 1

Interpréter ainsi l'ordre (par subdivision des classes) des partitions de $\mathrm{B}$ en termes d'ordre d'inégalité (et de concentration) des affectations, revient à compléter la Règle 1 par une Règle 1' : 
RÈGLE 1'. Tout transfert d'une partie des biens d'un "possédant" à un "non-possédant" diminue la concentration et l'inégalité.

En fait, cette Règle 1' est un cas particulier d'une règle plus générale (Règle 3 dite des transferts de Pigou et Dalton) que j'introduirai plus loin ; j'y reviendrai donc le moment venu. Pour l'instant, retenons que la Règle 1 qui postule l'équivalence entre individus conduit à un ordre très naturel pour l'inégalité et la concentration des affectations : celui des partitions (synonyme : classifications; nous sommes en terrain bien connu par la Psychologie génétique) de l'ensemble $\mathrm{B}$ des biens partagés.

\section{TROISIEME ETAPE, DEUXIEME REGLE : LES DISTRIBUTIONS (REPARTITIONS) ET LEUR SIMPLEXE}

Subdiviser les parts diminue l'inégalité ; encore faut-il que ces parts soient divisibles.

Un exemple non moins classique que le précédent : si l'on veut faire une répartition "égalitaire" d'un ensemble de trois biens constitué d'un chapeau, d'un chameau et d'un château, on sera bien en peine. Sauf à remplacer chacun de ces trois biens par sa valeur en nombre d'unités d'un autre bien, la monnaie, de sorte que l'affectation portera sur un ensemble B de biens (francs, ou dollars, ou yens, etc...) tous équivalents entre eux, interchangeables.

Et c'est ce que l'on fait toujours lorsque l'on doit procéder à une affectation ; dans le cas d'un héritage, on vendra le château, voire le chameau, si aucun des héritiers n'a de quoi régler aux autres la soulte lui permettant de le recevoir. Faire ainsi, c'est, du point de vue de la comparaison de l'inégalité des affectations, postuler une deuxième règle, duale de la première :

RÈGLE 2. L'inégalite d'une affectation (fonction de B dans I) est invariante par rapport au groupe des permutations de $\mathrm{B}$.

Remarquons que contrairement à la première règle (invariance pour les permutations de I) qui n'a été socialement admise (dans les sociétés occidentales) que très tardivement, cette deuxième règle correspond à la pratique de ces mêmes sociétés depuis des millénaires, en fait depuis l'invention de la monnaie. C'est un postulat qui quasiment "va de soi". Mais il n'en serait certes pas de même dans une société ignorant la monnaie.

Examinons les conséquences de cette condition en faisant pour l'instant, abstraction de la Règle 1, c'est-à-dire en considérant comme distincts, non interchangeables, les individus constituant l'ensemble I.

Ce qui compte maintenant dans une affectation, ce ne sont plus les biens particuliers attribués à chaque individu de I, mais seulement le nombre de ceux-ci ; la suite des effectifs des classes de la partition induite dans B par l'affectation, qui est, rappelons-le, une fonction de B dans I.

Posons : $\quad I=\{1,2, \ldots, \mathrm{i}, \ldots, \mathrm{k}\}$

La distribution (on dit encore : la répartition) d'une affectation est la suite (le vecteur) :

$$
\mathrm{x}=\left(\mathrm{x}_{1}, \mathrm{x}_{2}, \ldots, \mathrm{x}_{\mathrm{i}}, \ldots, \mathrm{x}_{\mathrm{k}}\right)
$$


où $\mathrm{xi}_{\mathrm{i}}$ est le nombre d'unités de biens attribuées à l'individu $i$; $\mathrm{xi}_{i}$ est donc un entier positif ou nul pour tout $i$, et la somme des xi est égale à $n$, effectif de l'ensemble $\mathrm{B}$ :

$$
\mathrm{Yi}, \mathrm{x}_{\mathrm{i}} \geq 0 ; \cdots \mathrm{x}_{\mathrm{i}}=\mathrm{n}=\mathrm{PBP}
$$

On démontre aisément que le nombre des affectations admettant la même distribution $\left(\mathrm{x}_{1}\right.$, $\left.\mathrm{x}_{2}, \ldots \mathrm{xi}_{1}, \ldots, \mathrm{x}_{\mathrm{n}}\right)$ est donné par l'expression dite "multinomiale" :

$$
\frac{\mathrm{n} !}{\mathrm{x}_{1} ! \mathrm{x}_{2} ! \ldots \mathrm{x}_{\mathrm{k}} !}=\frac{\left(\square \mathrm{x}_{\mathrm{i}}\right) !}{\square\left(\mathrm{x}_{\mathrm{i}} !\right)}
$$

Quant à ces distributions elles-mêmes, on peut les organiser naturellement selon un principe qui n'est pas étranger à notre objet : deux distributions seront dites contiguës si l'on passe de l'une à l'autre par transfert d'une unité de bien d'un individu à un autre. Si par exemple, il y a six unités de bien réparties entre trois individus, la distribution $(3,2,1)$ est contiguë à $(2,2$, $2)$, mais aussi à $(3,1,2)$ qui elle-même est contiguë à $(4,0,2)$, etc. La Figure 2 représente le réseau des contiguïtés pour les 28 distributions de six unités entre trois individus (sur la Figure, on a omis parenthèses et virgules pour alléger l'écriture $)^{4}$.

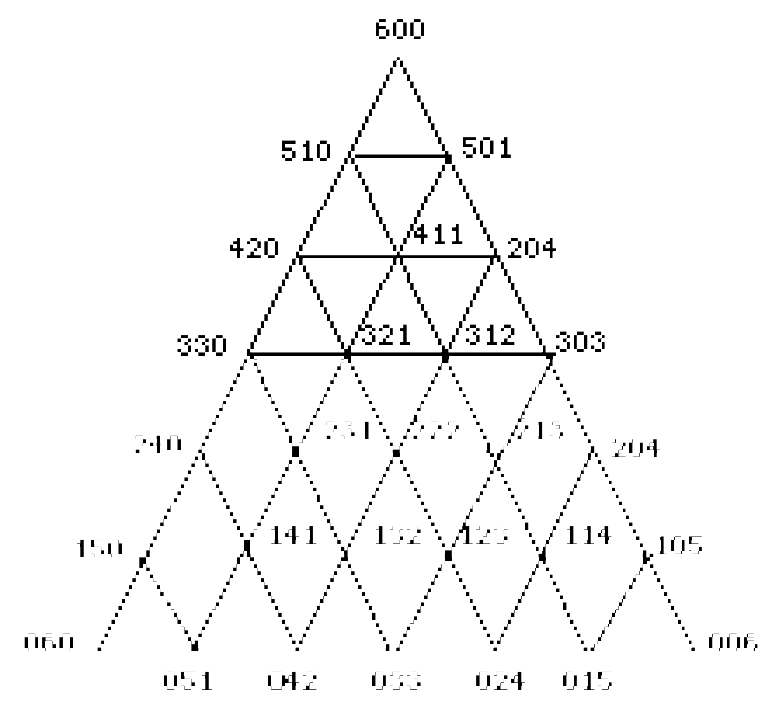

Figure 2

C'est un réseau en triangle, le simplexe $\mathrm{S}_{6,3}$; si l'on note $\mathrm{S}_{\mathrm{n}, \mathrm{k}}$ le simplexe des distributions (de $n$ unités de bien entre $k$ individus) organisées par contiguïté, les simplexes $\mathrm{S}_{\mathrm{n}, 2}$ sont figurés par des segments (Figure 3) ; les $\mathrm{S}_{\mathrm{n}, 3}$ on vient de le voir, par des triangles ; les $S_{n, 4}$ par des tétraèdres (Figure 4); les $S_{n, 5}$ par des "tétraèdres généralisés" (Figure 5) etc. Et les $S_{n, 1}$ par un seul point.

\footnotetext{
${ }^{4}$ Il semble que l'idée de représenter les répartitions à trois composantes par un triangle puisse être attribuée à d'Alembert (cf. R. Suaudeau, Les représentations figurées des physiocrates, Paris, Sirey, 1958, p.73 et sq.). Le commerce fournit d'ailleurs du "papier triangulaire" dont les graduations vont de 0 à $n=100$.
} 


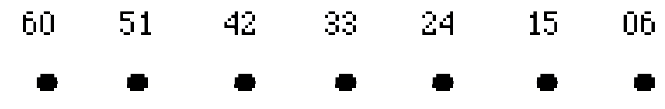

Figure 3

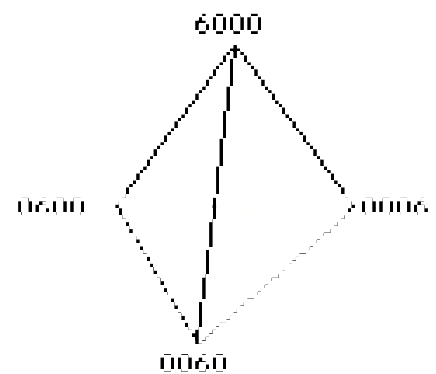

Figure 4

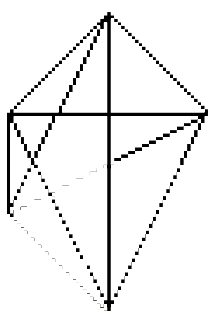

Figure 5

Il y a :

$$
\left|S_{n, k}\right|=\frac{(n+1)(n+2) \ldots(n+k-1)}{(k-1) !}=\frac{(n+k \square 1) !}{n !(k \square 1) !}
$$

distributions de $n$ biens entre $k$ individus (nombres "binomiaux"). Chacune est représentée par l'un des noeuds du diagramme en réseau, dont la méthode de construction est simple : on choisit dans l'espace de dimension ( $\mathrm{k}-1$ ) (de dimension 2, le plan, pour $\mathrm{k}=3$ ) $k$ points linéairement indépendants, les sommets du simplexe. Chacun est représentatif de l'un des individus constituant l'ensemble I ; et l'on gradue l'espace par les hyperplans de dimension ( $\mathrm{k}$ - 2) (les droites pour $\mathrm{k}=3$ ) $\mathrm{x}_{\mathrm{i}}=\mathrm{c}^{\mathrm{te}}$, la constante prenant les valeurs entières $0,1,2, \ldots, n$ (Figure $6)$.

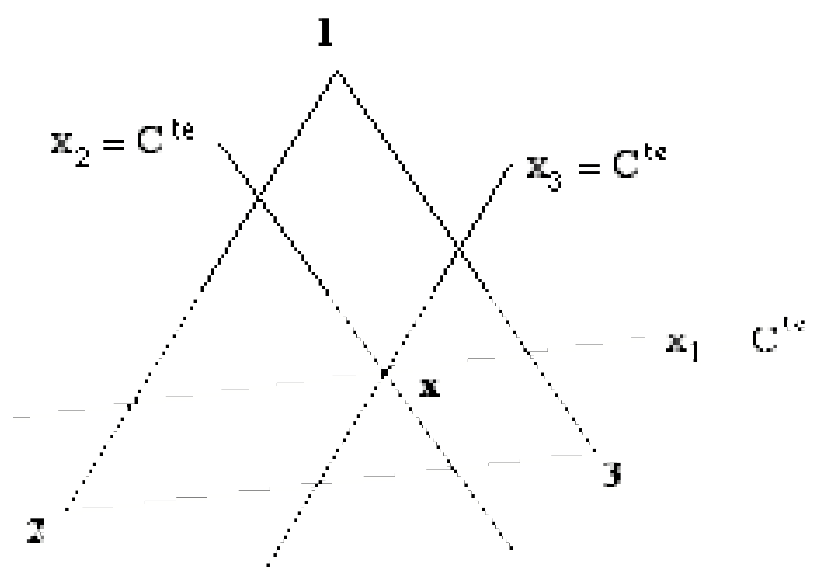

Figure 6

Bien entendu, cette représentation géométrique reste valide pour des valeurs réelles quelconques des $\mathrm{x}_{\mathrm{i}}$; le point

$$
\mathrm{x}=\left(\mathrm{x}_{1}, \mathrm{x}_{2}, \ldots, \mathrm{x}_{\mathrm{i}}, \ldots, \mathrm{x}_{\mathrm{k}}\right)
$$

avec $\mathrm{x}_{\mathrm{i}} \geq 0, \square \mathrm{x}_{\mathrm{i}}=n$, représente alors une distribution quelconque du total $n$ sur l'ensemble $\mathrm{I}$.

Du point de vue qui nous intéresse, celui de l'inégalité des distributions, il est clair que les sommets représentent les distributions d'inégalité maximale, dans lesquelles un seul individu reçoit la totalité des biens. 
De même, les points d'une arête telle que 12 (Figure 7), par exemple représentent les distributions pour lesquelles les deux seuls individus 1 et 2 se répartissent la totalité des biens, les autres n'ayant rien ; une face plane telle que 234 les distributions pour lesquelles seuls les individus 2,3 et 4 ont une part positive, etc. L'intérieur du simplexe est représentatif des distributions dans lesquelles aucun individu n'est réduit à la portion congrue d'une part nulle, et en particulier son barycentre $\mathrm{G}$ (le point 222 de la Figure 2, pour $n=6, k=3$ ) l'équirépartition dans laquelle tous les individus ont la même part. La topologie du simplexe nous fournit ainsi une typologie très claire des distributions : au voisinage des sommets, par exemple, on est proche des distributions de concentration maximale ; et au voisinage du barycentre, on est proche de l'équirépartition.

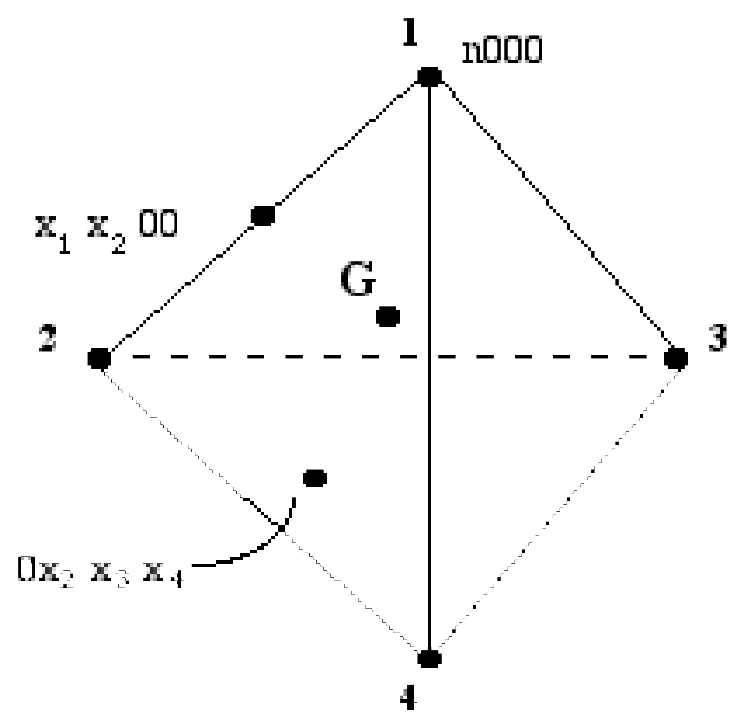

Figure 7

Celle-ci correspond-elle pour autant l'affectation la plus égalitaire?

Pas encore : la considérer comme telle, cela postulerait non seulement la Règle 2 (équivalence des biens) - que nous appliquons actuellement - mais aussi la Règle 1 (équivalence des individus), que nous avons provisoirement abandonnée. Nous y revenons maintenant.

\section{QUATRIEME ETAPE, TROISIEME REGLE : LES PARTAGES ET LEURS COURBES DE CONCENTRATION}

Si l'on postule simultanément les deux Règles 1 et 2, l'inégalité d'une affectation $\mathrm{f}$ : B 2 I est invariante à la fois par rapport au groupe des permutations de I, et par rapport à celui des permutations de B. Il n'y a donc plus à retenir désormais de chaque affectation que l'ensemble des parts de biens reçues par les individus : le partage du nombre $\mathrm{n}=$ "PBP en $\mathrm{k}=$ " $\mathrm{PI} \cdot \mathrm{P}$ parts 5 .

Ainsi, les six distributions $(3,2,1),(3,1,2),(1,3,2)$, etc., toutes constituées des mêmes parts 3,2 et 1 correspondent à un seul et même partage que nous écrirons par convention 321 (on va de la plus grande part à la plus petite ; on aurait pu aussi bien prendre la convention

\footnotetext{
${ }^{5}$ Attention. Dans la littérature anglo-saxonne, et parfois aussi en franglish, hélas, le même mot partition désigne ces deux concepts bien différents et à bien distinguer que sont la partition (classification) d'un ensemble et le partage d'un nombre.
} 
duale), ou, sous forme cumulée 356 : un seul individu a 3 unités du bien, à deux, ils cumulent 5 unités, et à trois la totalité des 6 unités. Les autres individus éventuels n'ont rien.

Le diagramme de la Figure 8 résume les relations entre les quatre objets fondamentaux de la construction : affectations, partitions, distributions, partages.

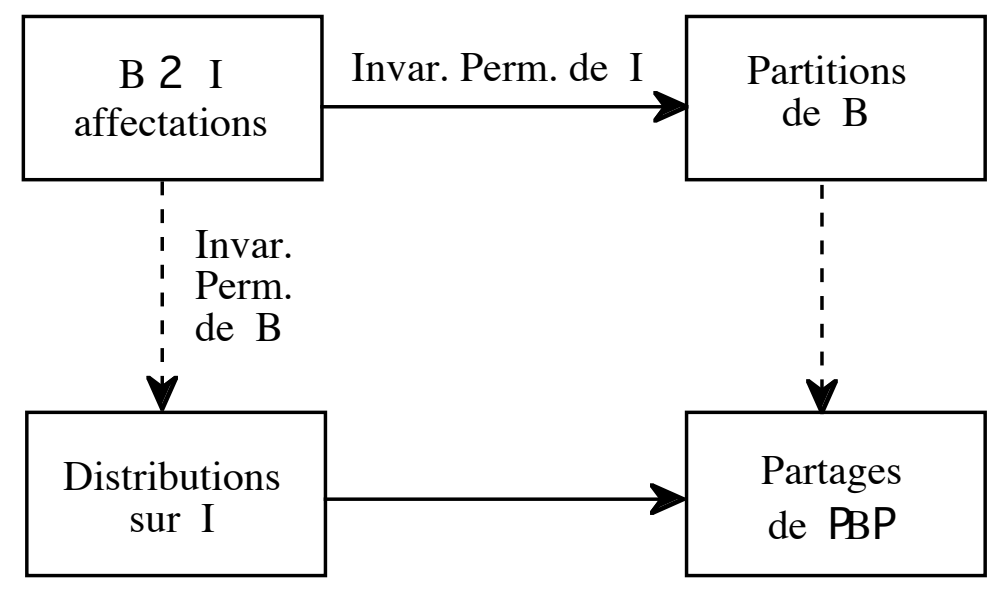

Figure 8

Remarque : à titre d'exercice, le lecteur pourra montrer ceci : pargageons l'entier $\mathrm{n}$ en $\mathrm{k}$ parts égales à $\mathrm{n}_{1}, \mathrm{n}_{2}, \ldots \mathrm{nh}_{\mathrm{h}}$ (avec $\mathrm{n}_{1}>\mathrm{n}_{2}>\ldots>\mathrm{n}_{\mathrm{h}} \geq 0$ ), et désignons par $\mathrm{k}_{1}, \mathrm{k}_{2}, \ldots, \mathrm{kh}$ le nombre de parts égales $\mathrm{n}_{1}, \mathrm{n}_{2}, \ldots, \mathrm{n}_{\mathrm{h}}$ respectivement. On a ainsi :

$$
\begin{aligned}
& \mathrm{k}=\mathrm{k}_{1}+\mathrm{k}_{2}+\ldots+\mathrm{k}_{\mathrm{h}}=\square \mathrm{k}_{\mathrm{j}} \\
& \mathrm{n}=\mathrm{n}_{1} \mathrm{k}_{1}+\mathrm{n}_{2} \mathrm{k}_{2}+\ldots+\mathrm{n}_{\mathrm{h}} \mathrm{k}_{\mathrm{h}}=\square \mathrm{n}_{\mathrm{j}} \mathrm{k}_{\mathrm{j}}
\end{aligned}
$$

Alors ce partage est l'image de :

$$
\begin{array}{ll}
\frac{\left(\square \mathrm{n}_{\mathrm{j}} \mathrm{k}_{\mathrm{j}}\right) !}{\square\left(\mathrm{n}_{\mathrm{j}} !\right)^{\mathrm{k}_{\mathrm{j}}} \mathrm{k}_{\mathrm{j}} \text { ! }} & \text { partitions d' un ensemble de } \mathrm{n}=\square \mathrm{n}_{\mathrm{j}} \mathrm{k}_{\mathrm{j}} \text { éléments } \\
\frac{\left(\square \mathrm{k}_{\mathrm{j}}\right) !}{\square\left(\mathrm{k}_{\mathrm{j}} !\right)} & \text { distributions d' un simplexe } \mathrm{S}_{\mathrm{n}, \mathrm{k}} \text { de } \mathrm{k}=\square \mathrm{k}_{\mathrm{j}} \text { sommets } \\
\text { et enfin de } & \\
\frac{\left(\square \mathrm{k}_{\mathrm{j}}\right) !\left(\square \mathrm{n}_{\mathrm{j}} \mathrm{k}_{\mathrm{j}}\right) !}{\square\left(\mathrm{n}_{\mathrm{j}} !\right)} & \text { affectations d' un ensemble de } n \text { biens à } k \text { individus. }
\end{array}
$$

Il nous faut maintenant ordonner les partages d'un point de vue qui sera celui de l'inégalité (ou de la concentration) par définition de ce dernier terme "inégalité d'un partage".

Le principe qui y préside, et qui va nous conduire à la troisième règle, procède à la fois de celui qui permet d'ordonner les partitions (par subdivision des classes) et d'organiser les distributions en simplexe (par transfert d'une unité de bien d'un individu à un autre). Si ce transfert d'une unité est fait d'une part $\mathrm{n}_{\mathrm{i}}$ à une part $\mathrm{n}_{\mathrm{j}}$ inférieure $\left(\mathrm{n}_{\mathrm{i}}>\mathrm{n}_{\mathrm{j}}\right)$, alors ou bien le partage n'est pas modifié (si $\left.n_{i}-n_{j}=1\right)$, ou bien il l'est $\left(s i n_{i}-n_{j} \geq 2\right)$, et on dira que l'inégalité du partage en a été diminuée. En itérant de tels transferts de "possédants" à "moins 
possédants", on obtient progressivement des partages de plus en plus égalitaires ; en particulier, si le transfert a lieu d'une part positive (et supérieure à 1) à une part nulle, on retrouve l'ordre par subdivision pour les partitions correspondantes.

Le diagramme de la Figure 9 représente ainsi l'ordre d'inégalité pour tous les partages de six biens entre six individus ; on a figuré à un même niveau tous les partages ayant même nombre de parts non nulles et sur une même "verticale" tous les partages ayant la même plus grande part ; chaque partage a été écrit sous les deux formes : suite des parts non nulles (première ligne), cumul des parts (seconde ligne). On chemine ainsi, dans le diagramme, du partage le plus inégalitaire (une seule part) au plus égalitaire, l'équirépartition des six biens entre six individus.

Remarque. Soit $\mathrm{p}_{\mathrm{n}, \mathrm{m}}$ le nombre des partages de $\mathrm{n}$ dont la plus grande part vaut $\mathrm{m}$, et toutes les parts sont strictement positives ; $\mathrm{p}_{\mathrm{n}, \mathrm{m}}$ est également le nombre de partages de $\mathrm{n}$ en $\mathrm{m}$ parts toutes strictement positives (le démontrer).

On $\mathrm{a}: \mathrm{p}_{\mathrm{n}, 1}=\mathrm{p}_{\mathrm{n}, \mathrm{n}}=1$

$$
\mathrm{p}_{\mathrm{n}, \mathrm{m}}=0 \text { si } \mathrm{m}>\mathrm{n} \text {. }
$$

Les nombres $p_{n, m}$ satisfont à l'équation de récurrence :

$$
\mathrm{p}_{\mathrm{n}, \mathrm{m}}=\prod_{\mathrm{i}=1}^{\mathrm{m}} \mathrm{p}_{\mathrm{n}-\mathrm{m}, \mathrm{i}}
$$

Sur les partages, cf. par exemple [8].

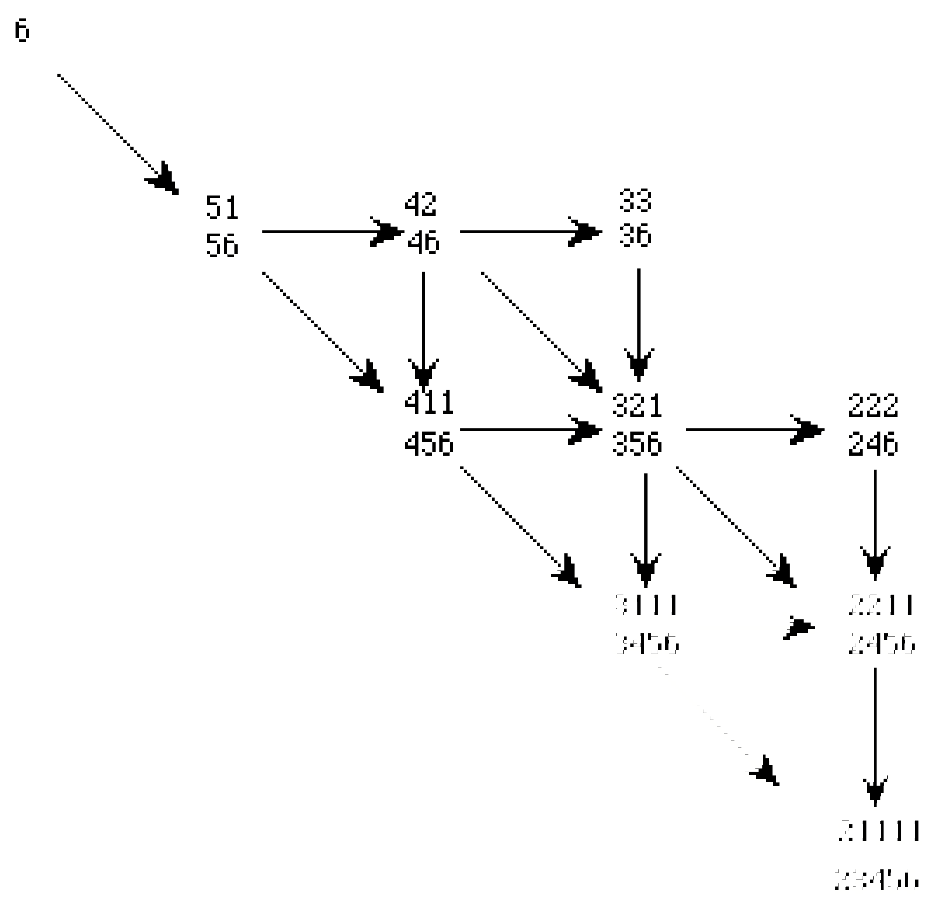


L'ordre d'inégalité entre affectations d'un ensemble $\mathrm{B}$ à un ensemble I donnés est ainsi défini par l'ensemble de trois règles : les Règles 1 et 2 d'invariance par rapport aux permutations des individus et des biens respectivement, et la Règle 3 :

RÈGLE (Axiome) 3. Tout transfert d'une unité de bien d'une part à une part inférieure diminue l'inégalité du partage (ou éventuellement la laisse invariante).

Cette règle est connue dans la littérature économique sous le nom de principe des transferts de Pigou et Dalton, du nom des deux économètres britanniques qui l'énoncèrent semble-t-il pour la première fois en 1920 sous une forme légèrement différente, mais équivalente.

On remarquera sur la Figure 9 que l'ordre d'inégalité est partiel (il n'est total que pour n $\leq 5)$; il est impossible, par exemple, de passer du partage 33 au partage 411 ou inversement par une suite de transferts de "riche" à "moins riche". Une représentation graphique très parlante des partages, et de leur ordre d'inégalité, est fournie par leurs courbes de concentration de Lorenz et Gini (M.O. Lorenz, économiste américain, utilisa le premier cette représentation, pour l'inégalité de distribution des revenus, vers 1905-1906 ; Corrado Gini en fit ultérieurement un usage abondant).

Donnons-nous deux axes de coordonnées (Figure 10), l'un pour l'ensemble I des invidus, l'autre pour celui B des biens; et représentons dans ce système les partages cumulés (en interpolant linéairement, pour la clarté de la lecture).

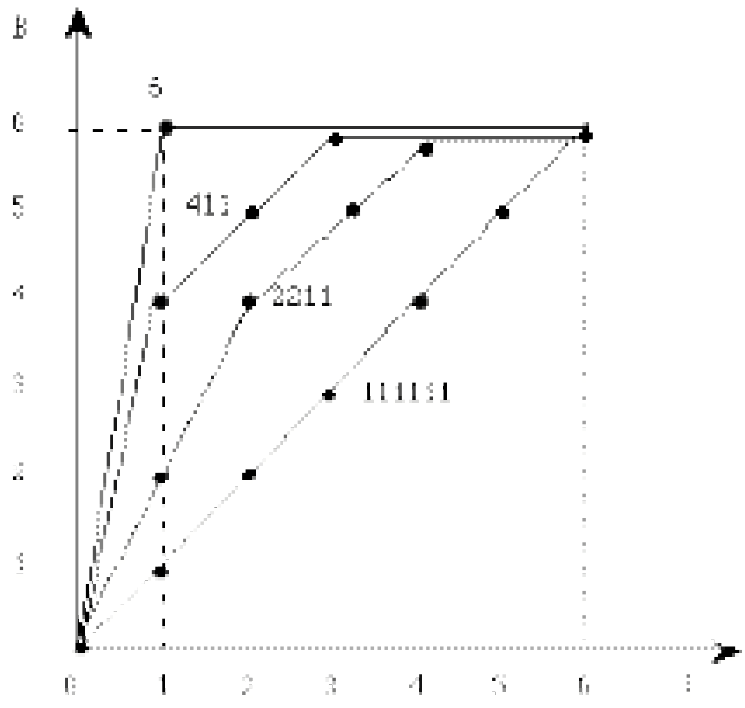

Figure 10

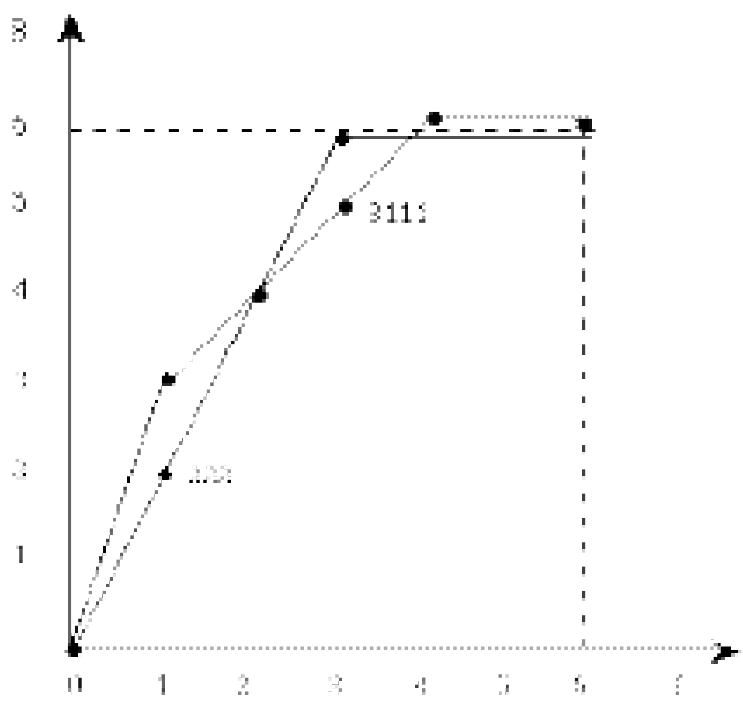

Figure 11

Chaque partage est ainsi figuré par une courbe convexe, linéaire par morceaux, sa courbe de concentration, dont la Figure 10 montre quelques exemples : un partage, tel par exemple que 2211, moins inégalitaire qu'un autre (411, par exemple), a sa courbe entièrement au dessous, majorée par celle de 411.

Le partage le plus inégalitaire a une courbe qui majore toutes les autres ; le plus égalitaire, celui de l'équirépartition, a pour courbe la bissectrice des axes. Lorsque deux partages sont non comparables, leurs courbes de concentration se coupent (Figure 11 pour les partages 3111 et 222 de 6). 
Remarque : certains économistes affaiblissent la règle 3. Ils supposent la population divisée en une suite de classes telles que les "tranches de revenus", par exemple : les classes sont ordonnées et tous les individus d'une classe ont des parts supérieures à toutes les parts de ceux de la classe suivante, etc. L'affaiblissement de la règle 3 consiste alors à dire que les seuls transferts d'un individu à un autre qui diminuent l'inégalité sont ceux d'une classe à une classe moins riche. Les tranferts internes à une classe laissent l'inégalité invariante.

\section{CINQUIEME ETAPE, QUATRIEME REGLE : CONCENTRATION NORMEE}

Reste à franchir une dernière étape, si l'on veut comparer l'inégalité d'affectations quels que soient leurs ensembles $\mathrm{B}$ et $\mathrm{I}$, c'est-à-dire, compte tenu des règles précédentes, quels que soient les effectifs de ces ensembles.

Il est clair en effet que le partage 222 de six unités de bien est assez inégalitaire s'il se fait entre 6 individus (dont 3 n'ont rien), alors qu'il est le plus égalitaire de tous les partages possibles entre trois individus seulement. On ramènera donc tant les individus que les parts qu'ils reçoivent à des proportions, des pourcentages du total : c'est là la quatrième (et dernière) règle.

Précisons. Soient $\mathrm{n}_{1}, \mathrm{n}_{2}, \ldots, \mathrm{n}_{\mathrm{h}}$ les valeurs des parts, avec $\mathrm{n}_{1}>\mathrm{n}_{2}>\ldots>\mathrm{n}_{\mathrm{h}} \geq 0$; et $\mathrm{k}_{\mathrm{j}}$ le nombre d'individus ayant une part égale à $\mathrm{n}_{\mathrm{j}}$. Le total :

$$
\mathrm{n}=\underset{\mathrm{j}=1}{\mathrm{~h}} \mathrm{n}_{\mathrm{j}} \mathrm{k}_{\mathrm{j}} \text { de biens est partagé entre } \mathrm{k}=\underset{\mathrm{j}=1}{\mathrm{~h}} \mathrm{k}_{\mathrm{j}} \text { individus. }
$$

Et la proportion : $\mathrm{p}_{\mathrm{j}}=\frac{\mathrm{k}_{\mathrm{j}}}{\mathrm{k}}$ des individus se voit attribuer la proportion :

$$
\mathrm{q}_{\mathrm{j}}=\frac{\mathrm{n}_{\mathrm{j}} \mathrm{k}_{\mathrm{j}}}{\mathrm{n}} \text { du total est partagé. }
$$

La courbe de concentration normée est alors définie par la suite de ses sommets $\left(P_{j}, Q_{j}\right)$ :

$$
\begin{array}{cc}
\mathrm{P}_{1}=\mathrm{p}_{1} & \mathrm{Q}_{1}=\mathrm{q}_{1} \\
\mathrm{P}_{2}=\mathrm{p}_{1}+\mathrm{p}_{2} & \mathrm{Q}_{2}=\mathrm{q}_{1}+\mathrm{q}_{2} \\
\cdot & \cdot \\
\cdot & \cdot \\
\cdot & \cdot \\
\mathrm{P}_{\mathrm{j}}=\underset{\mathrm{i}=1}{\mathrm{j}} \mathrm{p}_{\mathrm{i}} & \mathrm{Q}_{\mathrm{j}}=\underset{\mathrm{i}=1}{\mathrm{j}} \mathrm{q}_{\mathrm{i}}
\end{array}
$$

Entre deux sommets $\left(\mathrm{P}_{\mathrm{j}}, \mathrm{Q}_{\mathrm{j}}\right)$ consécutifs, on interpole linéairement.

Cette courbe, linéaire par morceaux, est encore convexe, et sa signification en termes d'inégalité des affectations est claire : une proportion $\mathrm{P}_{\mathrm{j}}$ des individus détient la proportion $\mathrm{Q}_{\mathrm{j}}$ du bien distribué, et c'est là, par construction, le maximum de ce qu'une proportion égale à $\mathrm{P}_{\mathrm{j}}$ de la population des individus peut détenir ; tout autre regroupement des individus en proportion égale à $\mathrm{P}_{\mathrm{j}}$ aura un pourcentage inférieur ou au plus égal à $\mathrm{Q}_{\mathrm{j}}$ du bien distribué. 


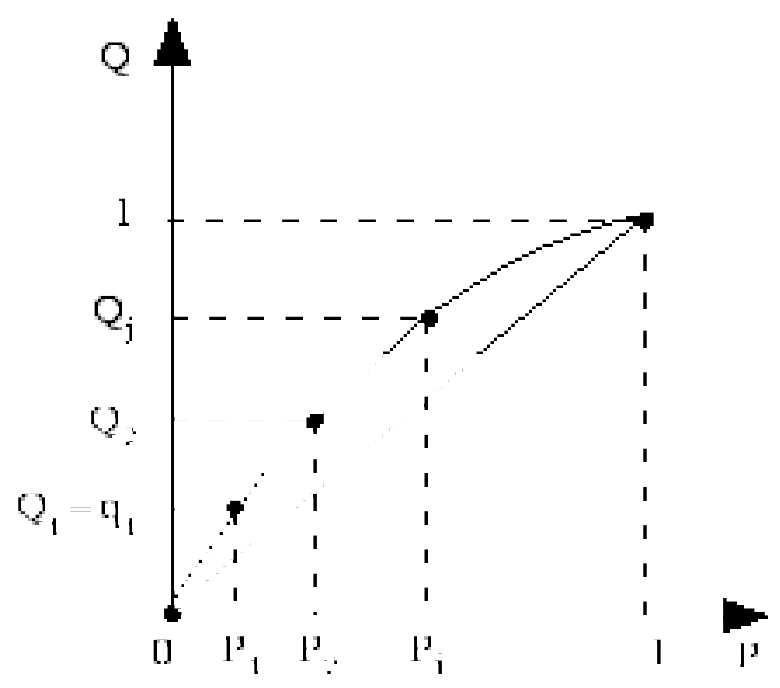

Figure 12

Finalement, l'ordre d'inégalité des affectations, c'est l'ordre de leurs courbes de concentrations normées. Etant données deux affectations : f : B 2 I et $\mathrm{f}^{\prime}$ : B' 2 I' on construit comme on l'a indiqué ci-dessus leurs courbes de concentration normées, $\mathrm{C}$ et $\mathrm{C}^{\prime}$ respectivement. Si C est entièrement au dessus de $C^{\prime}$ (ou, plus précisément, n'est jamais au dessous de $C^{\prime}$ ), l'affectation $\mathrm{f}$ sera dite par définition plus inégalitaire que $\mathrm{f}^{\prime}$ (Figure 13). Si par contre $\mathrm{C}$ et $\mathrm{C}^{\prime}$ se coupent, $\mathrm{f}$ et $\mathrm{f}^{\prime}$ sont non-comparables du point de vue de l'inégalité (Figure 14) ; notamment il existe alors un seuil $\left(\mathrm{P}_{0}, \mathrm{Q}_{0}\right)$ à partir duquel le sens, dans la comparaison des inégalités, se renverse. Il peut même y avoir plus de deux points d'intersection de ces deux courbes.

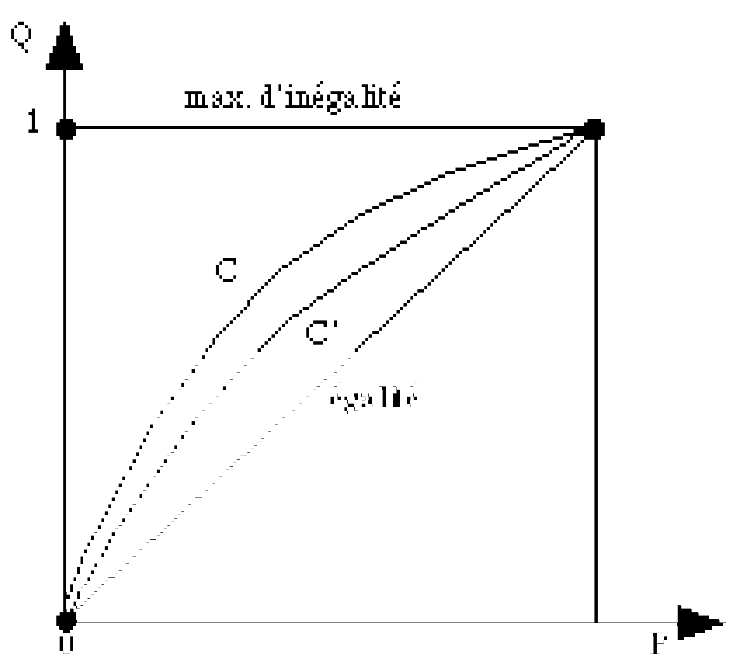

Figure 13

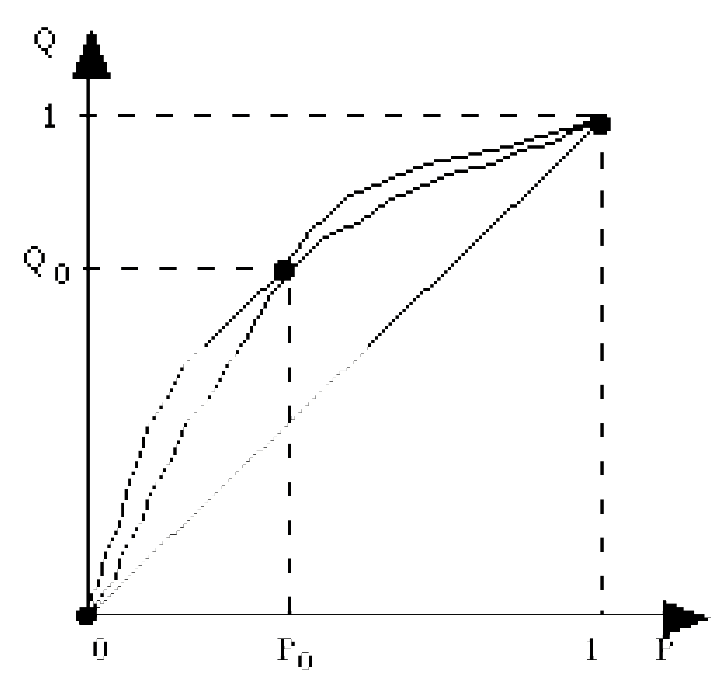

Figure 14

Les affectations les plus égalitaires (équirépartition) ont évidemment pour courbe de concentration normée la première bissectrice, et le maximum d'inégalité a pour courbe celle de la distribution de type "Dirac", qui concentre $100 \%$ de Q dès l'origine des P. 
Remrque. Etant donné deux distributions quelconques : $\overrightarrow{\mathrm{p}}=\left(\mathrm{p}_{1}, \mathrm{p}_{2}, \ldots, \mathrm{p}_{\mathrm{k}}\right) \quad \overrightarrow{\mathrm{q}}=\left(\mathrm{q}_{1}, \mathrm{q}_{2}, \ldots, \mathrm{q}_{\mathrm{k}}\right)$ avec $\square \mathrm{i}, \mathrm{p}_{\mathrm{i}} \geq 0, \mathrm{q}_{\mathrm{i}} \geq 0$ et $\underset{\mathrm{i}}{\square} \mathrm{p}_{\mathrm{i}}=\underset{\mathrm{i}}{\square} \mathrm{q}_{\mathrm{i}}=1$ on peut définir comme supra la courbe de concentration de $\overrightarrow{\mathrm{q}}$ par rapport à $\vec{p}$. Dans cette construction, $\vec{p}$ et $\vec{q}$ jouent des rôles dissymétriques. Diverses extensions de la notion de courbe de concentration, faisant jouer un rôle symétrique aux deux distributions, sont possibles. A ce sujet, voir par exemple [11] et [12].

\section{MESURER L'INEGALITE ? LES INDICES D'INEGALITE}

L'inégalité définie par les quatre règles qui ont été énoncées supra est donc une relation d'ordre mais celui-ci n'est que partiel ; il faut s'y résoudre, et ce d'autant plus que certains des axiomes qui fondent la notion peuvent, on l'a souligné, n'avoir aucun sens dans certaines situations réelles. Cette possibilité ne gène pas la plupart des statisticiens ou des économètres, parce qu'ils travaillent sur des données qui sont en général déjà mises sous la forme de distributions ou de partitions ; et les affectations qui en sont la source sont occultées.

Par contre, le psychologue, s'il monte - comme cela me semblerait souhaitable - des expérimentations sur la perception des inégalités et l'acquisition de leur comparaison se doit d'y être attentif. En statistique et en économétrie, on veut au contraire usuellement aller plus loin que la simple comparaison (partielle) des inégalités, et avoir une mesure de l'inégalité ; mesure, au sens du mesurage (measurement en anglais). On fabrique donc des indices (ou indicateurs) numériques de l'inégalité qui tous sont des mesures de l'écart de la distribution (pour laquelle l'indice est calculé) à l'équirépartition : plus cet écart est grand, plus l'inégalité est censée être forte. Géométriquement, ceci signifie, dans le simplexe (se reporter aux Figures 2 ou 7) que l'on calcule un écart du point représentatif de chaque distribution au barycentre du simplexe ; et pour les courbes de concentration, un écart de chaque courbe à la première bissectrice (voir. Figure $13)$.

Nombreux sont les indices utilisés ; les plus familiers d'entre eux ont nom khi-deux (ou $\square$ hi-deux), coefficient de variation (rapport de l'écart-type à la moyenne), écart moyen relatif (rapport de la somme des écarts absolus à la médiane au total partagé), entropie, coefficient de Gini (double de la surface comprise entre la courbe de concentration et la bissectrice). Ils ont une propriété commune, à laquelle doit satisfaire tout indice d'inégalité : celle d'être compatibles avec l'ordre partiel fourni par nos quatre règles, ou au moins par les trois premières, lorsque l'on travaille non sur les pourcentages, mais sur les nombres absolus. Compatibles, en ce sens que lorsque deux affectations ont des courbes de concentration normées comparables (comme sur la Figure 13), les valeurs correspondantes de l'indice doivent être dans le même sens. Par contre, ces divers indices, s'ils sont tous compatibles avec l'ordre partiel d'inégalité, ne sont pas toujours compatibles entre eux : tel indice peut croître alors que tel autre décroît, et c'est ce qui en rend le maniement très délicat. (Cf. par exemple [3] et [10]).

En fait, un indice d'inégalité n'a le plus souvent d'interprétation claire que lorsqu'il intervient comme paramètre d'une distribution théorique à laquelle s'ajustent les distributions observées, de la même façon que, par exemple, la moyenne et l'écart-type sont les deux paramètres des distributions de Laplace-Gauss. L'étude, du point de vue de leur inégalité, de distributions empiriques débouche nécessairement sur celle des distributions théoriques qui en rendent compte, et des "modèles" engendrant ces dernières (les principaux exemples sont les distributions Log-Normales de R. Gibrat (Cf. [1]) et les distributions de Pareto-Lévy (Cf. [4] ou [5]). Ceci n'est pas une surprise ; il en va toujours ainsi en statistique mathématique ; mais c'est un autre chapitre de celle-ci. 
La logique des inégalités, même dans ce qu'elle a de plus élémentaire, est moins facile à appréhender que celle de la classification ou même celle de l'espace. C'est probablement parce que les domaines auxquels elle se rapporte le plus souvent, ceux des inégalités économiques ou sociales, sont trop chargés de passion humaine pour que ces questions n'en soient pas obscurcies. Je suis néanmoins convaincu qu'elles pourraient être éclairées par des études de psychologie génétique convenablement conduites, et jouant notamment sur les quatre axiomes de base.

\section{POUR EN SAVOIR PLUS \\ BIBLIOGRAPHIE SOMMAIRE}

[1] ARMATTE, M., "Robert Gibrat et la loi de l'effet proportionnel", Math. Inf. Sci. hum., $\mathrm{n}^{\circ} 129,1995,5-35$.

[2] BARBUT, M., "Sur quelques propriétés élémentaires des fonctions de concentration de C. Gini", Math. Sci. hum., n 88, 1984, 5-19.

[3] BARBUT, M., "Sur les indicateurs de l'inégalité : croissance logistique et mesure de l'inégalité, et de quelques effets "paradoxaux" dans la comparaison des inégalités", Math. Sci. hum., $\mathrm{n}^{\circ} 90,1985,5-17$.

[4] BARBUT, M., "Distributions de type paretien et représentation des inégalités", Math. Inf. Sci. hum., n¹06, 1989, 53-69.

[5] BARBUT, M., "Une famille de distributions : des paretiennes aux contre-paretiennes. Application à l'étude de la concentration urbaine et de son évolution", Math. Inf. Sci. hum., $\mathrm{n}^{\circ} 141,1998,43-72$.

[6] BARBUT, M., MONJARDET, B., Ordre et classification. Algèbre et Combinatoire, Paris, Hachette, 1970, chap. VII.

[7] FOSTER, J.E., Inequality measurement, Proceedings of Symposium in Applied Mathematics, vol. 33, Providence, RI, American Mathematical Society, 1985, 31-68.

[8] GUILBAUD, G.Th., "Pour le deux cent cinquantième anniversaire de la mort de G.W. Leibniz. Un problème leibnizien : les partages en nombres entiers", Math. Sci. hum., n¹7, 1966, 13-56.

[9] RIORDAN, J., An introduction to combinatorial analysis, New York, Wiley, 1958.

[10] SEN, A., On Economic Inequality, London, Oxford-Clarendon Press, 1973.

[11] TEROUANNE, É., "Distorsion entre deux distributions d'une variable nominale", Math. Inf. Sci. hum., n¹31, 1995, 29-38.

[12] TEROUANNE, É., "Comparaison de tendances centrales par l'analyse des transferts", Math. Inf. Sci. hum., n¹34, 1996, 63-76. 